\title{
Procedimento Blupis e seleção massal em cana-de-açúcar
}

\author{
Ricardo Augusto de Oliveira (1*); Edelclaiton Daros ('); Marcos Deon Vilela de Resende $\left({ }^{2}\right)$; \\ João Carlos Bespalhok-Filho ('); José Luis Camargo Zambon ('); Tiago Rodrigues de Souza (3); \\ Ana Selena Fernandez Lucius ( $\left.{ }^{3}\right)$ \\ (') Universidade Federal do Paraná, Departamento de Fitotecnia e Fitossanitarismo, Rua dos Funcionários, 1540, 80035-050 \\ Curitiba (PR), Brasil. \\ (2) Embrapa Florestas, Estrada da Ribeira, Caixa Postal 319, 83411-000 Colombo (PR), Brasil. \\ (3) Universidade Federal do Paraná, Curso de Pós-graduação em Agronomia, Produção Vegetal, 80035-050 Curitiba (PR), Brasil. \\ $\left.{ }^{*}\right)$ Autor correspondente: rico@ufpr.br
}

Recebido: 23/fev./2011; Aceito: 2/ago./2011

\begin{abstract}
Resumo
O objetivo deste trabalho foi comparar a seleção via procedimento BLUP individual simulado (BLUPIS) versus seleção massal em famílias de irmãos-completos de cana-de-açúcar. Foram utilizadas 80 famílias originadas de cruzamentos biparentais da série RB03. O experimento foi desenvolvido em área experimental, localizado no Município de São Tomé, Paraná. O delineamento experimental foi em blocos incompletos, com cinco repetições por família. Para seleção considerou-se a variável tonelada de sólidos solúveis por hectare (TSH). Utilizou-se o modelo 35 do programa computacional Selegen REML/BLUP, via procedimento BLUPIS para a indicação do número de clones potenciais a serem selecionados. Estes resultados foram correlacionados com o número de clones selecionados via seleção massal dentro das famílias. A seleção clonal via procedimento BLUPIS indica maior número de clones promissores para caracteres quantitativos dentro de famílias com elevados efeitos genotípicos.
\end{abstract}

Palavras-chave: Saccharum spp., estratégias de seleção, REML/BLUP, BLUP indidividual simulado, modelos mistos.

\section{Blupis procedure and individual selection in sugarcane}

\begin{abstract}
The aim of this work was to compare the selection by simulated individual BLUP procedure (BLUPIS) versus individual selection in full-sib sugarcane families. Eighty families originated from bi crosses of RB03 series were used. The experiment was carried out in an experimental area in São Tomé County, Paraná State. The experimental design was in incomplete blocks, with five replications per family. For selection, the variable soluble solids per hectare (TSH) was used. For indication of the number of potential clones to be selected, the model 35 of the software Selegen REML/BLUP by BLUPIS procedure was used. The results of BLUPIS were correlated with the number of selected clones by individual selection. The results showed that clone selection by BLUPIS procedure can indicate higher number of promising clones for quantitative trait inside families with high genotypic effects.
\end{abstract}

Key words: Saccharum spp., selection strategies, REML/BLUP, simulated individual BLUP, mixed models.

\section{INTRODUÇÃO}

Uma das etapas mais importantes no melhoramento da cana-de-açúcar (Saccharum spp.) corresponde à fase inicial, denominada T1. Nesta fase, são produzidos milhares de indivíduos heterozigotos, provenientes de hibridaçóes entre genitores previamente selecionados (Cesnik e Miocque, 2004). Para esta fase, a seleção de famílias pode ser preferida quando a seleção é praticada com base em caracteres de baixa herdabilidade individual (FALCONER e MACKAY, 1996).
A seleção de famílias consiste em selecionar aquelas com elevados valores genotípicos, sendo descartadas famílias com valores genotípicos baixos. Estudos mostrando o potencial de famílias com valores genotípicos superiores, quando comparados com famílias de valores inferiores, evidenciam que a seleção com base nas melhores é efetiva para identificar quais teriam maior proporção de clones-elite. Neste caso, a seleção de famílias com base em caracteres quantitativos de produção, poderá possibilitar a identificação de clones promissores com maior probabilidade de serem mais produtivos. 
Esse processo representa uma vantagem quando a seleção de famílias é comparada com a seleção massal (KIMBENG e Cox, 2003).

$\mathrm{Na}$ seleção massal, também denominada seleção individual, as plantas são selecionadas com base em seus valores fenotípicos, pois, nesta fase não há repetição. Em cana-de-açúcar, na primeira fase de seleção, cada genótipo está repetido uma vez e em um único ambiente. Assim, a avaliação é visual e com base em características indiretas de produção. Com isso, a eficiência seletiva depende da quantidade de variabilidade existente na população-base a ser explorada, da herdabilidade do caráter a ser melhorado e da extensão do ganho genético deste caráter selecionado (Hogarth, 1971, Skinner, 1982; Bastos et al., 2003).

A seleção quando praticada em famílias com elevados valores genotípicos, pode possibilitar maior probabilidade de encontrar clones superiores em suas respectivas progênies (Barbosa et al., 2004; 2005; Resende e Barbosa, 2005, Oliveira et al., 2008). Atualmente, para o estudo de famílias de cana-de-açúcar tem se adotado o método dos modelos mistos REML/BLUP (REML é a máxima verossimilhança restrita, e BLUP, a melhor predição linear não viciada), que permite estimar os parâmetros genéticos e predizer os valores genotípicos das famílias (RESENDE, 2002a). Em cana-de-açúcar, a seleção de famílias na fase inicial do melhoramento, normalmente, é realizada considerando informaçóes totais das parcelas, pois elas são colhidas por inteiro. Para contornar esta situação, RESENDE e BARBosa (2006) propuseram a seleção via BLUP individual simulado (BLUPIS), que se baseia nos efeitos genotípicos das famílias avaliadas em campo.

O objetivo deste trabalho foi comparar a seleção via procedimento BLUP individual simulado (BLUPIS) versus seleção massal em famílias de irmãos-completos de cana-de-açúcar.

\section{MATERIAL E MÉTODOS}

O experimento foi desenvolvido em campo em área experimental de cana-de-açúcar, situada no Município de São Tomé, Região Noroeste do Estado do Paraná. A região está situada entre as coordenadas geográficas: $23^{\circ} 34^{\prime} 02,75^{\prime \prime}$ de latitude Sul e 52 $38^{\prime}$ '53,87" de longitude Oeste, e com altitude média de $450 \mathrm{~m}$, situada no Terceiro Planalto Paranaense e, de acordo com a classificação de Köeppen, possui clima tipo Cfa. O experimento foi instalado em solo tipo Latossolo Vermelho Distrófico, classificados como solos muito profundos, derivados de arenito Caiuá.

No estudo, foram utilizadas 80 famílias de irmãoscompletos, oriundos de cruzamentos biparentais realizados na Estação de Floração e Cruzamento da Serra do Ouro, Município de Murici, Alagoas, em 2003. As progênies foram plantadas e mantidas em casa de vegetação, localizada no município de Paranavaí, Estado do Paraná. As plântulas foram transplantadas para o campo em abril de 2004.

O delineamento experimental foi em blocos incompletos, com os tratamentos compostos pelas 80 famílias de irmãos-completos e a parcela experimental constituída de dez plantas dispostas em linha e espaçadas entre si em $0,5 \mathrm{~m}$. O espaçamento entre parcela/linha foi de $1,4 \mathrm{~m}$. $\mathrm{O}$ experimento foi colhido na cana-planta em abril de 2005, e a avaliaçáo, realizada no ciclo de cana-soca em abril de 2006. Para estimar a produtividade em tonelada de sólidos solúveis por hectare (TSH), consideraram-se as variáveis: número de colmos (NC), obtido pelo contagem do número total de colmos de cada touceira das parcelas experimentais, sendo posteriormente estimado para cada metro linear de sulco; diâmetro de colmos (DC), medido na base de cada colmo presente na parcela experimental com o auxílio de paquímetro, sendo posteriormente obtido a média de diâmetro de colmo por parcela; estatura dos colmos (EC), medido em todas as plantas da parcela com o auxílio de régua graduada considerando até a folha +0; teor de sólidos solúveis no colmo (\%), medido em três colmos de cada touceira presente na parcela experimental com auxílio de refratômetro de campo, sendo obtido a média por parcela. Com estes dados, foi feita a estimativa da massa de cada planta, em quilogramas, pela seguinte fórmula (Chang e Milligan, 1992):

$$
P C=\pi \times N C \times E C \times(D C / 2)^{2} x d
$$

$\mathrm{O}$ volume de um colmo foi considerado igual ao de um cilindro, e uma densidade populacional (d) foi de $1 \mathrm{~g} \mathrm{~cm}^{-3}$. A produção de cana por hectare $\left(\mathrm{TCH}, \mathrm{em} \mathrm{tha} \mathrm{e}^{-1}\right)$ foi estimada pela expressão: (PC x 10) / 0,7, onde 0,7 é a área ocupada pela planta $\left(\mathrm{m}^{2}\right)$. $\mathrm{O}$ rendimento em sólidos solúveis por hectare (TSH, em t ha-1) foi calculado pela equação (Leite et al., 2009):

\section{$T S H=(T C H x$ sólidos solúveis $) / 100$}

Os dados foram analisados via modelos mistos REML/ BLUP, cujo REML permitiu estimar os parâmetros genéticos e BLUP, predizer os valores genéticos aditivos e genotípicos. Este modelo estatístico está associado à avaliação de famílias de irmãos-completos obtidas sob cruzamento dialélico desbalanceado, no delineamento de blocos incompletos, com colheita total da parcela, considerando a equação matricial descrita abaixo, conforme RESENDE (2002a):

$$
y=X r+Z a+W p+S f+T b+e
$$

Em que: $y:$ vetor de dados; $r:$ vetor dos efeitos de repetição (assumidos como fixos) somados à média geral; $a$ : vetor dos efeitos genéticos aditivos individuais (assumidos como aleatórios); $p$ : vetor de efeitos de parcelas 
(aleatórios); $f$ : vetor dos efeitos genéticos de dominância associado a famílias de irmãos-completos (assumidos como aleatórios); $b$ : vetor dos efeitos dos blocos incompletos (aleatórios); $e$ : vetor de erros ou resíduos (aleatórios). $X, Z, W, S$, e $T$ representam as matrizes de incidência, respectivamente, para os efeitos de $r, a, p, f$, e $b$.

Para análise dos dados adotou-se o modelo 35, para dialelos não aparentados, do programa computacional Selegen REML/BLUP (Resende, 2002b). Este modelo fornece a indicação do número de indivíduos a serem selecionados dentro da cada família, pelo procedimento BLUP individual simulado (BLUPIS), conforme desenvolvido por ResEnde e BARBosa (2006).

Este procedimento estima de forma dinâmica o número $\mathrm{n}_{\mathrm{k}}$ de indivíduos a serem selecionados em cada família $k$, determinado por $n_{k}=\left(\hat{g}_{k} / \hat{g}_{j}\right) n_{j}$, em que $\hat{g}_{k}$ refere-se ao valor genotípico da família $k, \hat{\mathrm{g}}_{\mathrm{j}}$ refere-se ao valor genotípico da melhor família (número um da classificação) e $n_{j}$ equivale ao número de indivíduos selecionados na melhor família (Resende e Barbosa, 2005; 2006).

Conjuntamente, realizou-se a seleção massal no experimento contendo as 80 famílias, priorizando a seleção de genótipos para ciclo precoce de maturaçâo fisiológica. Neste caso, foram considerados os caracteres secundários que estáo correlacionados com a produtividade em açúcar por área, como: estatura de plantas, diâmetro de colmos, número de perfilhos por touceira e teor de sólidos solúveis, obtido com o refratômetro de campo. Nesta seleção considerou-se ainda o aspecto de touceira, sendo descartados genótipos com características indesejadas como a incidência de doenças, desuniformidade de perfilhos em estatura e diâmetro. Para determinar a precocidade dos genótipos, adotou-se o teor de sólidos solúveis das variedades-padráo (RB855156, RB855453) plantadas nas bordas experimentais, sendo os clones das famílias com mesmo teor de sólidos solúveis ou superior, considerados como potenciais para ciclo precoce no Estado do Paraná.

A correlação entre BLUPIS x Seleção Massal foi calculada a partir do número de genótipos potenciais indicados pelo procedimento BLUPIS e o número de genótipos obtidos na seleçấo massal, conforme descrito: $\mathrm{r}_{\text {blupis; massal }}=\operatorname{Cov}_{\text {(blupis;massal) }} \sigma_{\text {blupis }} \sigma_{\text {massal }}$; onde: $\operatorname{Cov}_{\text {(blupis;massal) }}$ : covariância entre blupis e seleção massal; $\sigma_{\text {blupis }}$ : desviopadrão pelo procedimento blupis; e $\sigma_{\text {massal }}$ : desvio-padrão para a seleção massal.

\section{RESULTADOS E DISCUSSÃO}

Os componentes de variância e os parâmetros genéticos estimados pelo REML/BLUP no estudo de 80 famílias de irmáos-completos estão apresentados na Tabela 1. Verificou-se que para o caractere TSH, a variação foi significativa (na Análise de Deviance) e explicada e pelo efeito genético aditivo dos parentais utilizados nas hibridaçôes, como também pelo efeito genético de dominância. Estes resultados indicam que os genitores com elevada performance são recomendados para serem utilizados em novos cruzamentos devido ao efeito genético aditivo, da mesma forma que se torna importante estudar grande número de cruzamentos para poder identificar as melhores famílias e realizar a seleção de clones dentro destas famílias em vista do efeito genético de dominância (BASTos et al., 2003).

Os coeficientes de variaçáo genética indicaram haver variabilidade com possibilidade de seleçáo para a produção de açúcar por hectare, representado pela variável TSH $(45,87 \%)$. Valores acima de $10 \%$, já indicam haver presença de variabilidade genética com possibilidade de seleção. A herdabilidade média da família no sentido amplo pode ser considerada de média magnitude $\left(\hat{\mathrm{h}}_{\mathrm{mf}}^{2}=0,56\right)$, o que permite selecionar famílias promissoras em produção de açúcar com boa acurácia seletiva entre as famílias $\left(\mathrm{Ac}_{\mathrm{fam}} 0,75\right)$, portanto, com adequada precisão entre os valores preditos e os valores verdadeiros (RESENDE, 2002).

A seleção das famílias promissoras para a variável TSH foi baseada na metodologia BLUPIS, proposta por RESENDE e BARbosa (2006), que permite a seleção considerando informaçóes totais das parcelas, pois as mesmas são colhidas totalmente. A seleção via BLUPIS permite determinar o número de indivíduos a serem selecionados dentro das famílias, o número total de indivíduos a serem selecionados e o número de famílias a serem selecionadas. Famílias com efeitos genotípicos negativos são eliminadas automaticamente, por estar abaixo da média geral do experimento, pois haveria baixa probabilidade de se obter

Tabela 1. Estimativa dos componentes de variância e parâmetros genéticos ${ }^{(1)}$, para produçáo em tonelada de sólidos solúveis por hectare (TSH), de 80 famílias de irmãos-completos de cana-deaçúcar, da série RB03. São Tomé (PR), 2006

\begin{tabular}{|cc|}
\hline Variável & Valor \\
\hline$\sigma_{\mathrm{a}}^{2}$ & 51,48 \\
\hline$\sigma_{\mathrm{bl}}^{2}$ & 6,27 \\
\hline$\sigma_{\mathrm{d}}^{2}$ & 9,94 \\
\hline$\sigma_{\mathrm{e}}^{2}$ & 113,55 \\
\hline$\sigma_{\mathrm{y}}^{2}$ & 181,24 \\
\hline$\hat{\mathrm{h}}_{\mathrm{a}}^{2}$ & $0,28 \pm 0,10$ \\
\hline$\hat{\mathrm{h}}_{\mathrm{g}}^{2}$ & 0,50 \\
\hline$\hat{\mathrm{h}}_{\mathrm{mf}}^{2}$ & 0,56 \\
\hline $\mathrm{Ac}$ & 0,75 \\
\hline $\mathrm{CV}$ & $0, \mathrm{~g} \%$ \\
$\mathrm{CVr}$ & 0,87 \\
\hline $\mathrm{MG}$ & 13,02 \\
\hline
\end{tabular}

(') Variância genética aditiva $\left(\sigma_{a}^{2}\right)$; variância entre blocos $\left(\sigma_{b l}^{2}\right)$; variância genética de dominância entre famílias $\left(\sigma_{\mathrm{d}}^{2}\right)$; variância residual entre parcelas $\left(\sigma_{\mathrm{e}}^{2}\right)$; variância fenotípica individual $\left(\sigma_{\mathrm{v}}^{2}\right)$; herdabilidade individual no sentido restrito $\left(\hat{\mathrm{h}}_{\mathrm{a}}^{2}\right)$; herdabilidade individual no sentido amplo $\left(\hat{\mathrm{h}}_{\mathrm{g}}^{2}\right)$; herdabilidade em nível de médias de família no sentido amplo $\left(\hat{\mathrm{h}}_{\mathrm{mf}}^{2}\right)$; acurácia seletiva entre famílias $\left(\mathrm{Ac}_{\mathrm{fam}}\right)$, coeficiente de variação genética $\left(\mathrm{CV}_{\mathrm{g}} \%\right)$; coeficiente de variação relativa $\left(\mathrm{CV}_{\mathrm{r}}\right)$ e $(\mathrm{MG})$ média geral. 
um clone promissor dentro destas famílias (RESENDE e BARBOSA, 2005; 2006).

Com base nesta metodologia, verificou-se que o número total de indivíduos a serem indicados à seleção foi de 343, sendo selecionadas 29 famílias com efeitos genotípicos positivos (Tabela 2). Ao considerar apenas as cinco melhores famílias (RB825336 x SP80-1816, RB825458 $x$ RB855156, SP80-3280 x RB845257, RB83102 x RB855113, RB8495 x RB835089) verificou-se que o número total de indivíduos indicados foi de 154, o que representa aproximadamente $45 \%$ do total de indivíduos indicados pelo BLUPIS. Ao considerar as dez melhores famílias este porcentual chega a $64 \%$. Destaque para as famílias RB825336 x SP80-1816, RB825458 x RB855156, SP80-3280 x RB845257, RB83102 x RB855113, RB8495 x RB835089, RB835089 x RB867515, SP701143 x SP77-5181, RB8495 x RB8317, respectivamente, com 50, 34, 25, 23, 22, 19, 19 e 16 indivíduos indicados para a seleção. Considerando estas quatro famílias, observou-se que foram indicados 132 indivíduos.

$\mathrm{Na}$ seleção massal, o número total de indivíduos selecionados foi 758, e nas 29 famílias com efeitos genotípicos positivos foram selecionados 119 genótipos (Tabela 2). Nota-se, portanto, que o número total de indivíduos selecionados apenas nas famílias com efeitos genotípicos positivos foi inferior aos indicados na seleção via BLUPIS, lembrando que a seleçáo massal baseia-se em caracteres indiretos de produção, podendo levar a menor eficiência seletiva de indivíduos promissores para esta característica. Neste contexto, como a seleção massal é baseada no fenótipo e nos valores individuais, observou-se que grande parte dos genótipos foram obtidos nas famílias com efeito genotípico negativo (639 genótipos).

Ao propor a seleção BLUPIS, Resende e Barbosa (2006) relatam que 50 indivíduos a serem selecionados dentro da melhor família sejam suficientes para conter o melhor indivíduo da progênie para o caráter produtividade, com níveis próximos de $98 \%$ da representatividade máxima da família. Portanto, ao adotar a seleção massal verificou-se que na melhor família foram selecionados apenas nove indivíduos, diminuindo assim, a probabilidade de conter o melhor indivíduo desta família.

Ao comparar os procedimentos de seleção via BLUPIS com a seleçấo massal (Tabela 3), verificou-se uma correlação de $r=0,66^{* *}$. Esta correlação significativa comprova que as duas metodologias indicam resultados semelhantes, porém na seleção massal há tendência de selecionar maior número de indivíduos oriundos de cruzamentos abaixo da média experimental, como foi obtido para o TSH. A magnitude desta correlação depende principalmente da herdabilidade dos caracteres de seleçáo. Caracteres com valores de herdabilidade individual acima de 0,5 proporcionariam a mesma eficiência seletiva de indivíduos quando comparado com a seleção de famílias (RESENDE, 2002a). Os resultados
Tabela 2. Número de indivíduos a serem selecionados dentro das famílias $\left(\mathrm{n}_{\mathrm{k}}\right)$ via método BLUPIS (variável TSH) e Seleção Massal realizada em famílias de irmãos-completos de cana-de-açúcar, série RB03, São Tomé (PR), 2006

\begin{tabular}{|c|c|c|c|}
\hline Clas ${ }^{(1)}$ & $\begin{array}{c}\text { Famílias de irmãos- } \\
\left.\text { completos ( }+ \text { e } \delta^{\lambda}\right)\end{array}$ & BLUPIS $\left(n_{k}\right)\left(n_{j}=50\right)$ & MASSAL \\
\hline $1^{(2)}$ & RB825336 x SP80-1816 & 50 & 9 \\
\hline 2 & RB825458 x RB855156 & 34 & 6 \\
\hline 3 & SP80-3280 x RB845257 & 25 & 7 \\
\hline 4 & RB83102 x RB855113 & 23 & 6 \\
\hline 5 & RB8495 x RB835089 & 22 & 7 \\
\hline 6 & RB835089 $\times$ RB867515 & 19 & 8 \\
\hline 7 & SP70-1143 x SP77-5181 & 19 & 4 \\
\hline 8 & RB8495 x RB8317 & 16 & 2 \\
\hline 10 & RB735200 x SP77-5181 & 12 & 4 \\
\hline 11 & RB835054 x RB931555 & 12 & 2 \\
\hline 12 & RB813804 x RB845197 & 11 & 4 \\
\hline 13 & RB925211 x RB8495 & 11 & 8 \\
\hline 14 & RB912850 x SP80-1816 & 10 & 7 \\
\hline 15 & RB855156 x SP80-1816 & 9 & 2 \\
\hline 16 & RB966928 x RB835054 & 9 & 6 \\
\hline 17 & RB855511 x SP80-1816 & 7 & 6 \\
\hline 18 & RB835089 x RB8495 & 7 & 4 \\
\hline 19 & SP83-2847 x RB956911 & 7 & 2 \\
\hline 20 & SP83-5073 x RB867515 & 6 & 2 \\
\hline 21 & RB855156 x RB925354 & 6 & 2 \\
\hline 22 & RB93509 x SP77-5181 & 6 & 3 \\
\hline 23 & C062-175 x RB92579 & 5 & 2 \\
\hline 24 & SP70-2233 x TUC7742 & 5 & 2 \\
\hline 25 & RB8317 x RB925345 & 5 & 4 \\
\hline 26 & RB768647 x RB92579 & 3 & 4 \\
\hline 27 & SP83-2874 x RB855463 & 2 & 1 \\
\hline 28 & RB855453 x SP83-2847 & 1 & 2 \\
\hline 29 & RB75126 x RB865463 & 1 & 3 \\
\hline Total & & 343 & 119 \\
\hline
\end{tabular}

(') Classificaçâo das famílias com base no $n^{\circ}$ de genótipos selecionados pelo procedimento BLUPIS;

() Consideraram-se apenas as famílias com valores genotípicos acima da média experimental para TSH;

$\mathrm{n}_{\mathrm{j}}=50$, número de indivíduos selecionados dentro da melhor família: $\mathrm{n}_{\mathrm{k}}=\left(\hat{\mathrm{g}}_{\mathrm{k}} / \hat{\mathrm{g}}_{\mathrm{l}}\right) 50$; $\hat{\mathrm{g}}_{\mathrm{k}}$ refere-se ao valor genotípico predito da família $\mathrm{k}$ e $\hat{\mathrm{g}}_{\mathrm{l}}$ ao valor genotípico da melhor família (número 1 da classificação)

Tabela 3. Estimativa do coeficiente de correlação entre o procedimento BLUPIS e a Seleção Massal, a partir de 80 famílias de irmãos-completos de cana-de-açúcar, da série RB03. São Tomé (PR), 2006

\begin{tabular}{|c|c|}
\hline Correlação (r) ${ }^{(1)}$ & $0,66553 * *$ \\
\hline Coincidência de indivíduos ${ }^{(2)}$ & 0,34694 \\
\hline $\begin{array}{l}\text { Proporção de indivíduos não } \\
\text { identificados na Seleção Massal (3) }\end{array}$ & 0,65708 \\
\hline \multicolumn{2}{|c|}{$\begin{array}{l}\text { (1) Correlação entre procedimento BLUPIS e a Seleção Massal. } \\
\text { (2) Número de indivíduos coincidentes entre BLUPIS e Seleçấo Massal. } \\
\text { (3) Proporção de indivíduos indicados pelo BLUPIS que não foram identificados na } \\
\text { Seleçáo Massal. } \\
\text { ** Significativo a } 1 \% \text { de probabilidade }(p<0,01) \text {. }\end{array}$} \\
\hline
\end{tabular}

presentes indicaram a vantagem da seleção de famílias para o caractere TSH, em que haveria ganhos significativos ao adotar esta metodologia de seleção. Ao comparar o número de indivíduos coincidentes em ambas as metodologias $(0,34)$ verificou-se que apenas $34 \%$ do número 
total de indivíduos indicados pelo BLUPIS foram selecionados também na seleção massal. Importante relatar que a seleçấo dentro das melhores famílias permitiria a seleção de genótipos com maior probabilidade e potencialidade para a produção de açúcar por área. Verificouse que o ganho de seleção com a seleção dos indivíduos dentro das 29 famílias indicou ser superior a 40\% $\left(19,4 t h^{-1}\right)$, em relação à média experimental.

Ao considerar os 758 genótipos selecionados na seleção massal dentro das 80 famílias, verificou-se que $84 \%$ destes foram oriundos de famílias com valores genotípicos abaixo da média experimental $\left(13,0 \mathrm{t} \mathrm{ha}^{-1}\right)$. Ressaltase ainda, que mais de $65 \%$ dos indivíduos indicados pelo BLUPIS não foram identificados na seleção massal (Tabela 3). Uma explicação para este baixo número de indivíduos selecionados dentro das melhores famílias seria decorrente da seleçáo massal ser realizada com base em características fenotípicas, sendo descartados indivíduos que possuíam sintomas de doenças. Das famílias estudadas, relata-se que no cruzamento entre RB825336 x SP80-1816 foi observada elevada incidência de carvão (Ustilago scitaminea), sendo um dos motivos que na seleção massal foram selecionados apenas nove indivíduos e que não tinham sintomas da doença.

Ao propor o procedimento BLUPIS, Resende e BARBosa (2006) relataram que a seleção via este procedimento permite alta correlaçấo entre os verdadeiros valores estimados via BLUP individual. A seleção, considerando as informaçôes das famílias, pode ser praticada quando os indivíduos estão sendo dirigidos para testes clonais via propagaçáo vegetativa. No caso da cana-deaçúcar, BLUPIS poderá proporcionar aumento na eficiência da seleção clonal, pois se baseia em caracteres quantitativos de produção o que permite selecionar famílias com clones promissores para estas características de produção.

\section{CONCLUSÃO}

A seleção clonal via procedimento BLUPIS permite identificar clones promissores para caracteres quantitativos, principalmente nas famílias com elevados efeitos genotípicos. O procedimento BLUPIS, quando comparado com a seleção massal possibilita maior eficiência em identificar maior número de genótipos promissores visando maior produção de sólidos solúveis por hectare.

\section{REFERÊNCIAS}

BARBOSA, M.H.P., RESENDE, M.D.V., BRESSIANI, J.A., SILVEIRA, L.C.I., PETERNELLI, L.A. Selection of sugarcane families and parents by Reml/Blup. Crop Breeding and Applied Biotechnology, v.5, p. 443-450, 2005.

BARBOSA, M.H.P.; RESENDE, M.D.V; PETERNELLI, L.A. BRESSIANI, J.A.; SILVEIRA, L.C.I.; SILVA, F.L.; FIGUEIREDO, I.C.R. Use of Reml/Blup for the selection of sugarcane families specialized in biomass production. Crop Breeding and Applied Biotechnology, v.4, p.218-226, 2004.

BASTOS I.T.; BARBOSA M.H.P.; CRUZ, C.D.; BURNQUIST, W.L.; BRESSIANI, J.A.; SILVA F.L. Análise dialélica em clones de cana-de-açúcar. Bragantia, v.62, p.199-206, 2003.

CESNIK, R.; MIOCQUE J. Melhoramento da cana-de-açúcar. Brasília: Embrapa Informação Tecnológica, 2004. 307p.

CHANG, Y.S.; MILLIGAN, S.B. Estimating the potential of sugarcane families to produce elite genotypes using univariate cross prediction methods. Theoretical and Applied Genetics, v.84, p.662-671, 1992.

FALCONER, D.S.; MACKAY, T.F.C. Introduction to quantitative genetics. 4.ed. London: Longman, 1996. 464p.

HOGARTH, D.M. Quantitative inheritance studies in sugarcane; II: correlations and predicted responses to selection. Australian Journal of Experimental Agriculture, v.22, p.103-109, 1971.

KIMBENG, C.A.; COX, M.C. Early generation selection of sugarcane families and clones in Australia: a review. Journal of the American Society of Sugar Cane Technologists, v.23, p.20-39, 2003

LEITE, M.S.O.; PETERNELLI, L.A.; BARBOSA, M.H.P.; CECON, P.R.; CRUZ, C.D. Sample size for full-sib family evaluation in sugarcane. Pesquisa Agropecuária Brasileira, v.44, p.1562-1574, 2009.

OLIVEIRA, R.A.; DAROS, E.; BESPALHOK-FILHO, J.C.; ZAMBON, J.L.C; IDO, O.T.; WEBER, H.; RESENDE, M.D.V.; ZENI-NETO, H.; Seleção de famílias de cana-de-açúcar via modelos mistos. Scientia Agraria, v.9, p.269-274, 2008.

RESENDE, M.D.V. Genética, biométrica e estatística: no melhoramento de plantas perenes. Brasília: Embrapa Informação Tecnológica, 2002a. 975p.

RESENDE, M.D.V. O software Selegen REML/BLUP. Colombo: Embrapa Informação Tecnológica, 2002b. 67p. (Documentos - 77)

RESENDE, M.D.V.; BARBOSA, M.H.P. Selection via simulated BLUP based on family genotypic effects in sugarcane. Pesquisa Agropecuária Brasileira, v.41, p.421-429, 2006.

RESENDE, M.D.V; BARBOSA, M.H.P. Melhoramento genético de plantas de propagação assexuada. Colombo: Embrapa Informação Tecnológica, 2005. 130p.

SKINNER, J.C. Efficiency of bunch-planted and single-planted seedlings for selecting superior families in sugarcane. Euphytica, v.31, p.523-537, 1982. 